\title{
Don et sciences sociales. Théories et pratiques croisées
}

E. MAGNANI (dir.), Don et sciences sociales. Théories et pratiques croisées, Dijon, EUD, 2007, 244 pages (coll. « Sociétés »)

\section{Eliana Magnani}

\section{OpenEdition}

12 Journals

\section{Édition électronique}

URL : https://journals.openedition.org/cem/8092

DOI : $10.4000 /$ cem. 8092

ISSN : 1954-3093

Éditeur

Centre d'études médiévales Saint-Germain d'Auxerre

\section{Édition imprimée}

Date de publication : 15 août 2008

ISSN : 1623-5770

Référence électronique

Eliana Magnani, «Don et sciences sociales. Théories et pratiques croisées », Bulletin du centre d'études médiévales d'Auxerre | BUCEMA [En ligne], 12 | 2008, mis en ligne le 09 juillet 2008, consulté le 22

septembre 2022. URL : http://journals.openedition.org/cem/8092 ; DOI : https://doi.org/10.4000/cem. 8092

Ce document a été généré automatiquement le 22 septembre 2022.

\section{(i) (ㅇ)

Creative Commons - Attribution - Pas d'Utilisation Commerciale - Partage dans les Mêmes Conditions 4.0 International - CC BY-NC-SA 4.0

https://creativecommons.org/licenses/by-nc-sa/4.0/ 


\section{Don et sciences sociales. Théories et pratiques croisées}

E. MAGNANI (dir.), Don et sciences sociales. Théories et pratiques croisées, Dijon, EUD, 2007, 244 pages (coll. « Sociétés ») ${ }^{1}$

\section{Eliana Magnani}

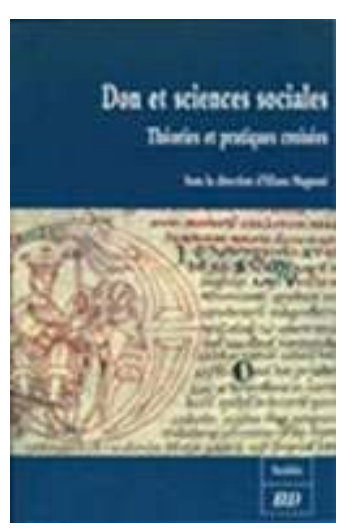

1 Les problèmes posés par le don comme catégorie d'analyse et à analyser en sciences sociales n'ont pas été résolus, comme l'atteste ce volume où s'expriment les voix, souvent discordantes, d'anthropologues, d'archéologues, d'historiens, d'historiens de l'art, de philosophes et de sociologues. Issu de la table ronde qui s'est tenue à Auxerre en janvier $2006^{2}$, ce livre se présente comme un échantillon de la critique et du débat actuels sur l'usage, la pertinence et les alternatives à la théorie maussienne, sur des voies nouvelles ou renouvelées de la réflexion théorique et de la recherche sur le don. Il croise des regards dissemblables et essaie d'amorcer ainsi le difficile, mais toujours stimulant, dialogue entre les disciplines du social.

2 La mise en perspective historique de nos concepts et notions est indispensable à ce type de démarche. La réception de la théorie du don, selon Marcel Mauss (1924), dans les sciences humaines et sociales, s'est faite le plus souvent sans recul historique. Ce phénomène généralisé est d'autant plus saisissant chez les historiens médiévistes qu'il est conjugué avec une sorte d'oubli, comme il est rappelé par Eliana Magnani dans le 
premier article de ce volume. Oubli de ce que Mauss a utilisé des notions forgées, dès le milieu du XIX ${ }^{e}$ siècle, par les historiens du droit et de la langue germaniques du haut Moyen Âge. C'est le cas notamment du "don-échange » ou du "don-contre-don » employés souvent de façon mécanique et réductrice dans la médiévistique depuis Georges Duby (1973). Les médiévistes ignorent souvent, et à l'exception de l'historiographie allemande, que le principe de réciprocité inhérent à la notion de doncontre-don (Gegengabe, Widergabe) apparaît dès 1848 dans une étude à caractère étymologique de Jacob Grimm. Reprise ensuite par Karl von Amira (1892) et Richard Meyer (1898), le «don-contre-don » avait déjà une longue histoire quand Mauss écrivit Essai sur le don.

3 Significativement, avant celui-ci, dans un article de 1921 sur « Une forme ancienne de contrat chez les Thraces ", Mauss concluait en souhaitant que des spécialistes réalisent l'étude « de la donation germanique et des échanges dans les textes celtiques». Son proche collaborateur, Henri Hubert, sociologue des religions et archéologue, a été le premier à y appliquer la théorie du don aux sociétés celtiques. Hubert fondait, cependant, son analyse sur les légendes et les mythes irlandais et gallois, connus dans leur version écrite seulement à partir du $\mathrm{x}^{\mathrm{e}}$ siècle de notre ère, et négligeait toutes les sources archéologiques, ainsi que les témoignages des auteurs classiques sur les Celtes. Comme le détaille Luc Baray, la mort prématurée d'Henri Hubert († 1927), associée à un rejet par l'archéologie française de tout ce qui pouvait passer pour théorique, ont fait que le concept du don n'est à nouveau employé dans les analyses des sociétés protohistoriques qu'en 1978, avec les travaux de Susan Frankenstein et Michael Rowlands, sur le système princier du premier âge du fer, repris et étendu ensuite à l'âge du bronze en 1987, par Patrice Brun dans son Princes et princesses de la Celtique. En retenant les formes agonistiques du don (potlatch) et en appliquant indistinctement aux sociétés celtiques le modèle dégagé pour les royautés africaines par Claude Meillassoux $(1960,1972)$, les analyses proposées par ces auteurs pêchent, entre autres, par la confusion grandissante établie entre les concepts de «système de don » et d' « économie de biens de prestige », pourtant bien distincts. Ils diluent ainsi la valeur heuristique que le don pourrait avoir dans la compréhension de ces sociétés, représentées par eux comme des sociétés toujours en crise, instables et dépendantes de l'économie-monde.

4 Pour ce qui est de la Grèce ancienne, l'essai de Mauss de 1921 sur les « festins » chez les Thraces, ne suscite pas d'échos jusqu'aux articles précurseurs d'Émile Benveniste sur "Le don et l'échange dans le vocabulaire indo-européen », et de Louis Gernet sur " La notion mythique de la valeur en Grèce », datés de 1948 tous deux. Ils restent pourtant méconnus jusqu'au milieu ou encore à la fin des années 1960. C'est à cette époque que l'ouvrage de Moses I. Finley, Le monde d'Ulysse (1954), très influencé par Karl Polanyi, est traduit en français. Avec Finley s'impose l'idée du don comme lieu de toutes les institutions de la Grèce archaïque. Plus largement, c'est par son biais que la notion de don-contre-don est introduite dans l'historiographie en général. En fait, le recours au don pour caractériser la société homérique, mais aussi les sociétés celtes ou la société du haut Moyen Âge, sert désormais d'élément classificatoire pour ceux qui, selon une perspective évolutionniste ou comparatiste, les considèrent comme des sociétés "primitives». C'est tout une autre perspective qu'adopte Paul Veyne (1976), en étudiant l'évergétisme ancien et en cherchant à bien le distinguer, au Bas-Empire, de la donation chrétienne. Le don ne reste pas moins l'une des clés d'interprétation de la 
société grecque, comme dans certains épisodes qui précédèrent les guerres médiques rapportés par Hérodote, où les échanges entre acteurs de statuts différents, les rois perses et leurs sujets, peuvent être envisagés en termes de "réciprocité inégale », dont nous parle dans ce recueil Évelyne scheid-Tissinier.

Dans le domaine de la représentation idéelle des échanges, la lecture de Mauss qu'a donnée Maurice Godelier (L'énigme du don, 1996) continue d'exercer une notable influence sur les travaux récents des médiévistes. La prise en compte du divin (surnaturel) comme acteur des rapports sociaux incite ainsi Tania Kambourova à interpréter, à nouveaux frais, des images trop vite considérées comme «illustration d'un acte pieux ou affirmation publique d'un acte politique ». La surface des fresques des églises (slavo-)byzantines du Moyen Âge est l'espace où " prend corps » le don surnaturel de la couronne aux souverains par la main droite de Dieu, par le Christ, par la Vierge ou par un saint, ainsi que le contre-don du souverain sous la forme d'un objet liturgique, d'une maquette d'église, d'une somme d'argent ou d'un acte de donation. L'image apparaît donc comme le lieu où se réalisent les échanges entre terre et ciel.

6 Saisies par Jean-Paul Desaive dans les archives auxerroises des XVII ${ }^{e}$ et XVIII ${ }^{e}$ siècles, les formes du don dans les modes de transmission patrimoniale ponctuent les différentes étapes du cycle de vie. Au moment de l'union matrimoniale, le contrat de mariage prévoit la succession lors de la disparition de l'un des conjoints. La démission des biens sert de contrepartie au soutien apporté au temps de la vieillesse et de la maladie. La donation entre vifs est, alors, surtout pratiquée entre les époux sans enfant. Enfin, le testament, dont la motivation première demeure d'ordre spirituel, fait une large place aux récompenses adressées aux serviteurs domestiques, tout en préservant le patrimoine des héritiers directs.

7 Mais qu'en est-il des sociétés où les pratiques assimilées au don ne sont pas aussi évidentes que dans ces sociétés historiques ? Dans le contexte interprétatif que domine toujours le paradigme anthropologique de la "prédation", l'analyse du rituel des esprits des animaux, chez les Miraña de l'Amazonie colombienne, engage Dimitri Karadimas à y lire les formes idéologiques de l'auto-justification des dominations - en l'occurrence ici, celle des humains sur les animaux. Le rituel des esprits des animaux, où les humains invitent les « Maîtres des animaux » en vue d'être accueillis ensuite dans la forêt, fait apparaître le don comme moyen de légitimation interne de l'acte de prédation : on donne, on n'attend pas de contrepartie, on prend. À l'équilibre du modèle du "don-échange " - donner, recevoir, rendre, où le donataire reçoit et rend -, il est possible d'opposer le déséquilibre inhérent au modèle du « donprédation » ou " don pour la prédation » - donner, recevoir, prendre, où le donateur donne et prend, dans un circuit où donner n'est pas la garantie de l'alliance et où le conflit peut surgir à tout moment. Une telle perspective encourage à nuancer la notion globalisante de prédation pour caractériser les sociétés amazoniennes, en prenant en considération des phénomènes qui renvoient aux représentations idéologiques de la réciprocité.

$8 \mathrm{Au}$ cœur de ces approches se pose le problème des notions mises en œuvre dans les interprétations historiques, archéologiques et anthropologiques, et, plus généralement, la place que ces disciplines occupent dans l'élaboration des concepts, surtout dans une situation "postmoderne " de "crise » des sciences sociales, avec l'abandon de l'idée d'une théorie sociologique générale, au profit d'un investissement dans la description de la diversité sociale. Dans ce contexte, le développement d'outils conceptuels souples, 
propres à rendre compte de la très grande multiplicité du don, pourrait conduire, en dernière instance, à l'élaboration d'une théorie plus générale et synthétique du don. C'est ce que propose Ilana Silber, autour de la métaphore sociologique de "répertoire " en la confrontant, et en la complétant, avec l'apport étonnamment sous-exploité de Paul Veyne dans Le pain et le cirque (1976), avec les avancées récentes du «tournant typologique", avec aussi l'idée de "registre du don" et l'étude de cas de Natalie Zemon Davis dans son Essai sur le don dans la France du XVI $I^{e}$ siècle (2000). L'enjeu d'un « répertoire » du don est la prise en compte et l'articulation aussi bien de la diversité que des contextes historiques dans lesquels s'insère le don.

Il importe aussi, dans tout cela, d'évaluer le legs de Mauss et de sa "découverte » du don - sorte d'hybride, mêlant intérêt et désintéressement, obligation et liberté - à déterminer l'effectivité ou pas de son universalité et de sa charge normative. Considérant le don comme une réalité spécifique, qui peut être expliquée par ellemême, propre à résoudre les paradoxes de l'existence humaine, Alain Caillé avance l'enquête empirique comme moyen d'organiser un système de questions en vue d'une théorie sociologique générale, le " paradigme du don et du politique (et symbolique)». Le don serait articulé autour de quatre pôles interdépendants, mais irréductibles entre eux : en tant qu'opérateur politique - le choix de l'alliance plutôt que la guerre et que la rivalité -, « reconnaisseur » d'identité qui façonne en même temps les sujets individuels et politiques; comme vecteur structurant des relations humaines, ou de la "socialité primaire » et, au-delà du politique, réalisé par devoir, par aimance [amour] ou par plaisir, avec sa part de liberté et de créativité. Le tout, en tenant compte des implications normatives et éthiques, dont nous sommes partie prenante, et qui étaient déjà les points conclusifs de Mauss dans son Essai. Une telle interprétation « irréductioniste » va à l'encontre des explications du don de type " économiciste »autour de l'idée de «profit»-, ou «inexistentialistes »- le don n'existe pas ou dans très peu de sociétés -, ou qui considèrent le don comme phénomène secondaire, incomplet, subordonné à une réalité plus essentielle - l'échange en général, le sacré, le symbolique, le sacrifice...

10 Le parti pris théorique, exposé par Alain Testart, emprunte une tout autre direction, critique et en rupture par rapport à la théorie maussienne : en insistant sur l'obligation du contre-don, Mauss aurait confondu don et échange - dans l'interprétation de la kula, par exemple. Faisant jouer entre elles des catégories juridiques, A. Testart définit le don comme une cession de bien qu'implique la renonciation de tout droit sur ce bien. Aucune contrepartie ne serait exigible. Ainsi défini, plutôt qu'un phénomène universel, le don serait une forme sociale qui peut assumer des expressions différentes dans les sociétés. C'est l'idée de départ d'une distinction, précisée ailleurs (Critique du don, 2007), entre «trois types de transfert ». Le don, premier type de transfert, serait par principe sans condition: même si un retour (un contre-don) peut être attendu, demandé ou sollicité, il ne peut pas être exigé. Dans le deuxième type, dont l'échange serait une composante, la contrepartie est exigible et obligatoire. Enfin, le troisième type de transfert, qui relève de l'état de dépendance sociale, «est exigible sans qu'aucune contrepartie ne le soit ».

11 Au lieu de la nier, François Athané opte ici pour tester l'hypothèse de l'universalité du don. Il emploie pour autant une approche naturaliste et comportementaliste associée au rapport à une règle et au langage. Si le don est un fait universel humain, il faut vérifier s'il est ailleurs dans la nature et se référer notamment aux travaux en éthologie 
et à la notion d'altruisme chez les animaux. La transmission des gènes ne passe pas seulement par la reproduction sexuée, mais aussi par un comportement altruiste qui favorise la reproduction d'individus apparentés. Le transfert de ressources dans l'espèce humaine contient une part de naturalité et une part de culture. Le comportement ne suffisant pas à définir le don, il faut y déceler une règle. Si le don est universel, il est lié à l'universalité d'une règle. Et celle-ci pourrait être le "don nourricier" des adultes envers le nourrisson, forme première de transfert de ressources.

12 La multiplicité d'interprétations et des discussions sur le don, présentées dans ce volume, témoignent de la difficulté des échanges entre disciplines, mais aussi de tout leur intérêt. Comme note Anita Guerreau-Jalabert dans l'article conclusif, les problèmes de compréhension entre chercheurs résident dans l'absence d'un cadre commun de réflexion reposant sur la comparaison de systèmes sociaux dans leur totalité. Ils résident aussi dans l'ignorance de l'évolution de la civilisation occidentale. La notion de don serait ainsi un héritage d'une histoire culturelle et sociale de l'Occident dont on ne peut pas faire l'abstraction. Au Moyen Âge, une théorie du don est élaborée par des penseurs chrétiens à partir d'une conception du lien social fondée sur l'amour (caritas) qui transite par Dieu et irrigue toute la société. La circulation des biens étant l'un des moyens à travers lequel les liens sociaux s'établissaient et se renouvelaient, le don était compris à l'intérieur de cette logique de "circulation généralisée » de la caritas. Entre le $\mathrm{XVII}^{\mathrm{e}}$ et le $\mathrm{xVIII}{ }^{\mathrm{e}}$ siècle, la séparation du religieux et de l'économique rompt avec les conceptions pluriséculaires d'un système de représentations médiéval fondé sur les valeurs du christianisme. Ce qui avait été de l'ordre du social devient alors de l'ordre du privé, transformé en valeur morale. C'est dans ce cadre particulier que s'enracinent les conceptions du don depuis le XIX ${ }^{e}$ siècle. Elles traversent les sciences sociales jusqu'à nos jours. Mais sans une perspective historique sérieuse et un effort convergeant vers un langage commun dans les disciplines du social, le débat autour du don demeure une question d'actualité, toujours irrésolue.

\section{NOTES}

1. Voir : « http://www.u-bourgogne.fr/EUD/don.html ». Nous reprenons ici, de façon légèrement remaniée, les propos développés dans l'introduction à l'ouvrage (p. 7-12). 2. E. MAGNANI, « Don et sciences sociales. Théories et pratiques croisées (compte rendu) », Bulletin du Centre d'études médiévales d'Auxerre, 10 (2006), p. 199-205 [publication électronique : «http://cem.revues.org/document383.html. »]. 
INDEX

Mots-clés : don 